\title{
Photoacoustic microscopy: a novel approach for studying perforator skin flap in a mouse model
}

\author{
Dong Zhang, Hairen Chen, Xiang Hu, Aixi Yu \\ Department of Orthopedics Trauma and Microsurgery, Zhongnan Hospital of Wuhan University, Wuhan, China
}

Contributions: (I) Conception and design: A Yu, X Hu, D Zhang; (II) Administrative support: A Yu; (III) Provision of study materials or patients: A Yu; (IV) Collection and assembly of data: D Zhang, H Chen; (V) Data analysis and interpretation: D Zhang; (VI) Manuscript writing: All authors; (VII) Final approval of manuscript: All authors.

Correspondence to: Aixi Yu, Xiang Hu. Zhongnan Hospital of Wuhan University, 169 East Lake Road, Wuhan, Hubei 430072 China.

Email: yuaixi@whu.edu.cn; 1534836434@qq.com.

Background: A comprehensive understanding of cutaneous microvessels is key to the design and use of the perforator skin flap. Compared with the various imaging technologies that have been applied in the clinical practice of the perforator skin flap, photoacoustic microscopy (PAM) is a very promising noninvasive imaging modality with high resolution and deep penetration in biological tissues.

Methods: PAM was employed to explore its multiple applications in a perforator skin flap. The following experiments were then conducted in 3 parts. In part 1, 7 mice were used to obtain the preoperative perforator mapping on the mouse back. In parts 2 and 3, 7 mice were used to design and harvest the multiterritory perforator flap. The status of the flap and the morphological changes of choke vessels were subsequently observed by PAM at several time points.

Results: The results showed that PAM could visualize and assess the vascular physiological and pathological conditions of the skin tissue in real time in vivo with high spatial and temporal resolution. It could also provide preoperative perforator mapping, including the total number of perforators, localization, vascular territories, and diameter. Furthermore, it could offer a quantitative, objective method to monitor the status of the perforator skin flap, and was capable of noninvasive characterization of the changes of choke vessels that play an important role in multiterritory perforator skin flap expansion and survival.

Conclusions: PAM has great potential to be an effective and precise quantitative imaging tool for perforator skin flap research, such in as flap design, monitoring, and choke vessel observation.

Keywords: Photoacoustic microscopy; perforator skin flap; choke vessel

Submitted Feb 06, 2021. Accepted for publication May 07, 2021.

doi: 10.21037/qims-21-135

View this article at: https://dx.doi.org/10.21037/qims-21-135

\section{Introduction}

A perforator flap is a flap that includes skin and subcutaneous tissue, which is perfused by a cutaneous perforator that perforates muscle or fascia. For decades, perforator flap surgery has been widely used in the field of repair/reconstructive surgery to conceal skin and soft tissue defects due to trauma or following tumor extirpation by local or distant transfer (1). Compared with other types of flaps, perforator flaps can achieve the aim of optimal reconstruction with minimum donor-site morbidity and shortened operative time through design of the flap based on the perforator location as well as according to donorand recipient-site needs with no damage to source vessels (2).

However, perforators are very small and fragile blood vessels, and there is often significant variation in the diameter, anatomical location, number, origin, and 
hemodynamic features in different individuals (3). Each perforator has its own vascular boundary (known as the perforasome) whose multidirectional flow pattern is complex and unique (4). Thus, it is crucial for surgeons to reduce complications by determining the number, localization, vascular boundary, and caliber of perforators before they design and harvest the perforator flaps in the clinical area. Also, postoperative vascular complications, such as thrombosis, pedicle twisting, and vasospasm, could lead to flap failure by impairing the oxygen and nutrient supply (5). Early and quantitative assessment of the flap status and prompt surgical intervention are key contributors to successful flap salvage when complications do arise. In addition, when faced with large and complex defect reconstruction, a single perforasome may not be suitable for this situation, and therefore, it is necessary for surgeons to explore to use the multiterritory perforator flaps involving several perforasomes (6-8).

In the cutaneous circulation, each perforasome is linked to the adjacent perforasomes through a series of reduced-caliber vessels that Taylor and Palmer called choke vessels, which allow a perforator to supply beyond its own perforasome $(4,9)$. Thus, choke vessels make it feasible to harvest larger perforator flaps by incorporating neighboring perforasomes into the flap based on an individual perforator, such as multiterritory perforator flaps. Yet, the clinical use of multiterritory perforator flaps is often hindered by distal partial necrosis occurring at the adjacent choke vessel boundary, which is caused by poor understanding of the choke vessels that play an indispensable role in flap expansion and survival (10). To our knowledge, the current approaches to the morphological study of the choke vessels cannot realize the aim of providing noninvasive, dynamic, and in vivo observation. Therefore, there is a pressing need for a reliable technique that reflects the actual physiological and pathological behaviors of choke vessels in living animals.

However, despite this need for a technique that reliably reflects the true physiological and pathological behaviors of cutaneous circulation, effective imaging modalities for studying perforator flaps remain largely limited. Nowadays, the most frequently used imaging tools for perforator flap design, monitoring, and choke vessel observation are ultrasound, $\mathrm{X}$-ray angiography, magnetic resonance angiography (MRA), and computed tomographic angiography (CTA). However, these modalities exhibit certain intrinsic limitations: (I) current approaches cannot completely achieve the aim of providing noninvasive, dynamic, in vivo, real-time observation; (II) their resolution is still unsatisfactory to resolve the microvasculature; and (III) some modalities require the injection of angiographic agents, which may disturb the intrinsic physiology of the microcirculation and hinder longitudinal monitoring (10-14). Given the challenges faced by the currently available imaging modalities for studying perforator flaps, the development of new imaging modalities that provide non-invasive, high-resolution, and comprehensive characterization of the cutaneous microvasculature in perforator flap has attracted considerable attention.

As a novel no-invasive biomedical imaging modality based on endogenous contrast from hemoglobin, photoacoustic microscopy (PAM) offers great potential to fill these technological deficiencies (15). PAM works based on the photoacoustic effect, and combines the features of high resolution of pure optical imaging and high penetration depth of ultrasound imaging. After a specific wavelength laser pulse is delivered into tissue, the energy is partly absorbed by hemoglobin and converted into heat, which leads to a transient thermoelastic expansion of red blood cells, thereby generating the broadband frequency ultrasound known as photoacoustic signals $(16,17)$. These photoacoustic signals are detected and converted into electrical signals via an ultrasonic transducer. Subsequently, these electrical signals are obtained by the data acquisition system and finally analyzed to produce computer-generated vessel images (18). Due to the fact that the scattering of ultrasonic waves in tissues is far less than that of light, it is easier for PAM to acquire high-resolution images in a noninvasive way compared to pure optical imaging or ultrasound imaging. Compared to conventional imaging technologies, PAM benefits from its high resolution, deep penetration, noninvasiveness, simplicity, time- and cost-efficiency, etc., and has developed into a significant imaging modality in the study of cerebrovascular disorders, tumor angiogenesis, skin burns, port wine stains, and various vascular complications (19-22).

The aim of this study was to describe this novel imaging technology for studying perforator skin flaps in terms of obtaining preoperative vascular mapping information, monitoring the flap tissue status, and observing the morphological changes of choke vessels, which would facilitate better clinical use of perforator skin flaps.

\section{Methods}

\section{PAM system}

The schematic of the PAM imaging system is shown in 

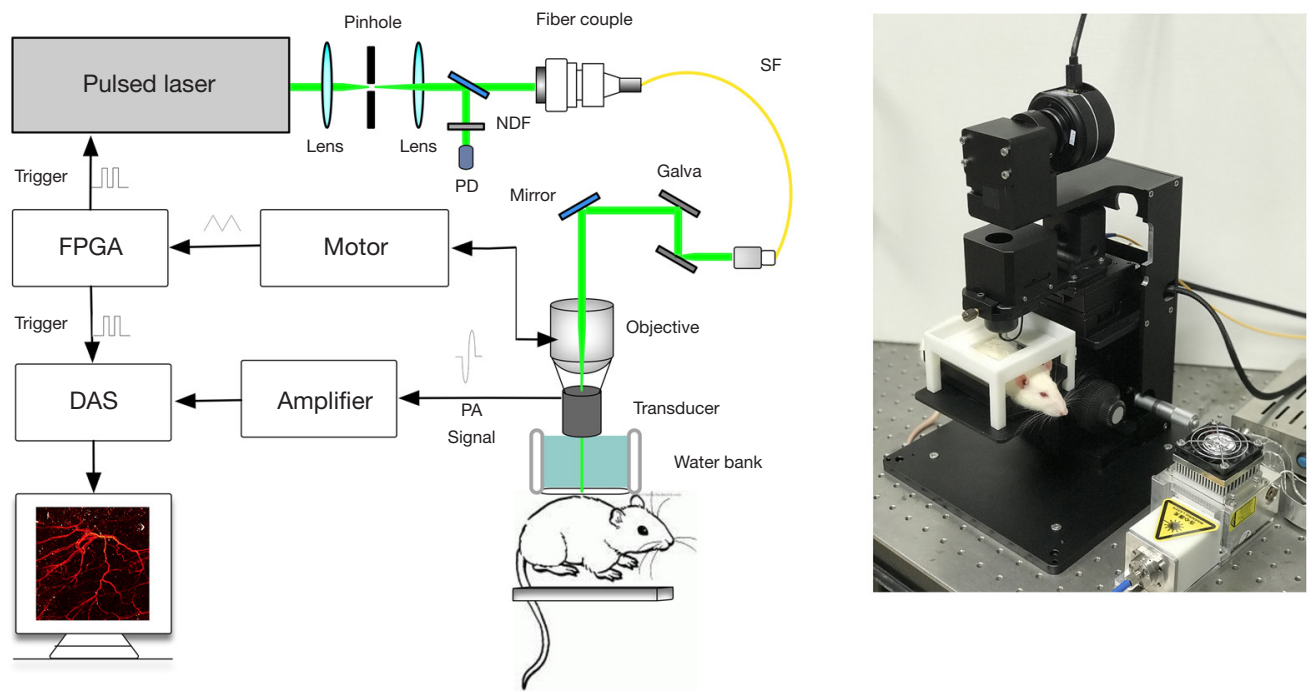

Figure 1 Schematic of the photoacoustic imaging system. NDF, neutral density filter; PD, photodiode; SF, single-mode fiber; FPGA, field programmable gate array; DAS, data acquisition system.

Figure 1. A miniature laser (532-2-V, Innolas, Germany), which operates a wavelength of $532 \mathrm{~nm}$ with a pulse width of $12 \mathrm{~ns}$ and a repetition rate of $50 \mathrm{kHz}$, was used as the irradiation source. The laser beam was coupled into a singlemode fiber (460HP, Thorlab, USA) via a fiber collimator (PAF-X-7-A, Thorlab, USA). The laser beam from the fiber was then collimated and focused by a fiber collimator (F240FC-532, Thorlabs, USA) and 4 multiples objective (GCO-2111, Daheng Optics, China) to excite the sample. The energy density on the tissue surface was approximately $11 \mathrm{~mJ} / \mathrm{cm}^{2}$, which is below the American National Standards Institute (ANSI) safety limit of $20 \mathrm{~mJ} / \mathrm{cm}^{2}$ (23).

\section{Animal preparation}

All efforts were made to minimize animal suffering. Our experimental protocol was approved by the Committee on the Ethics of Animal Experiments of Wuhan University (approval no. 2019173). We purchased female BALB/c mice (aged 5-6 weeks, weight 20-30 g) from the Laboratory Animal Center of Wuhan (China). Prior to photoacoustic imaging, the region of interest was shaved and chemically depilated with a depilatory cream. All surgical procedures and PAM measurements were performed under general anesthesia with ketamine hydrochloride $(30 \mathrm{mg} / \mathrm{kg}$ body weight), and $2 \%$ lidocaine gel was used around the incision to relieve the postoperative pain.
Visualization of the perforator mapping on the dorsal skin of rats

To obtain preoperative perforator mapping, seven mice were used for PAM imaging. For mouse-back imaging, the region of interest was divided into right and left borders on the right and left anterior axillary line, the cranial border on the suprascapular line, and the caudal border parallel to the iliac crest, respectively. The area is shown in Figure $2 A$. The PAM image in the entire region of interest was obtained (Figure 2). The detailed information was marked with arrows using the imaging tools.

\section{Observation of the flap status and changes of the choke region in the multiterritory perforator skin flap model}

To quantitatively assess the perforator flap status and observe the vascular morphological changes of the choke region via PAM, 7 mice were used in this part of the study. The mice were placed in the lateral decubitus position, and the upper and lower limbs were stretched slightly and fixed. The multiterritory perforator skin flaps were harvested at a level beneath the panniculus carnosus, which was distally based on the deep circumflex iliac perforator and included the intercostal perforasome in sequence. The perforator flaps were then sutured in back in their original positions using a nylon 5-0 suture (Figure 3).

To quantitatively assess the perforator flap status, PAM 


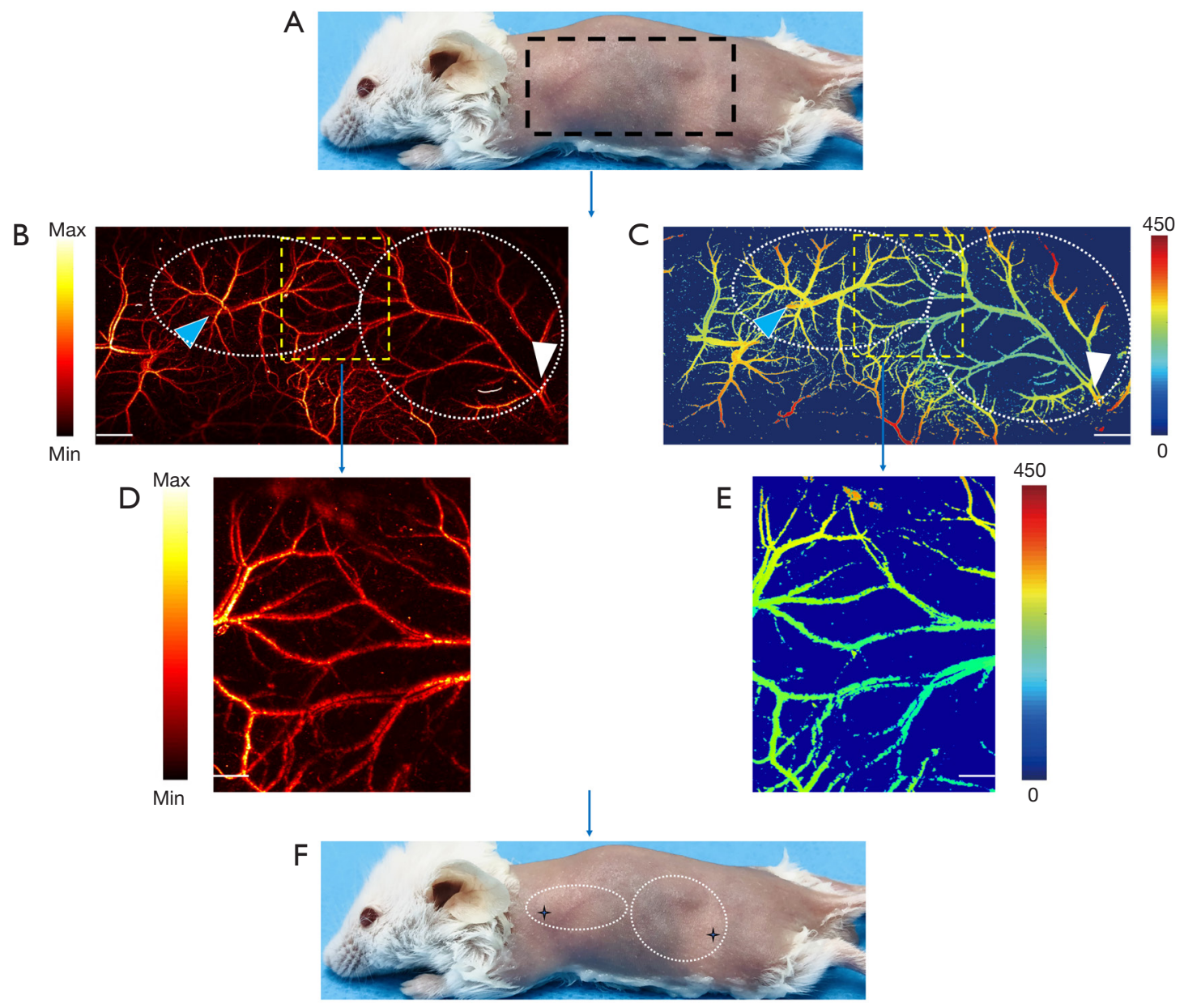

Figure 2 The digital and PAM images of the mouse back. (A) The planning area of the mouse back for photoacoustic microscope imaging. (B) In vivo PAM imaging of the vascular morphological mapping in the mouse back. (C) The depth-encoded image of B. (B,C) The blue arrow indicates an intercostal perforator, and the white arrow indicates a deep circumflex perforator; the white dotted line shows the vascular territories of the intercostal perforator and deep circumflex perforator. (D) The choke zone between the deep circumflex iliac perforator and the intercostal perforator. (E) The depth-encoded image of D. (F) The perforators' vascular territories and location data were transferred to the back skin. The black arrow indicates the PAM-based perforator locations. The white dotted line indicates the vascular territories of the perforators.
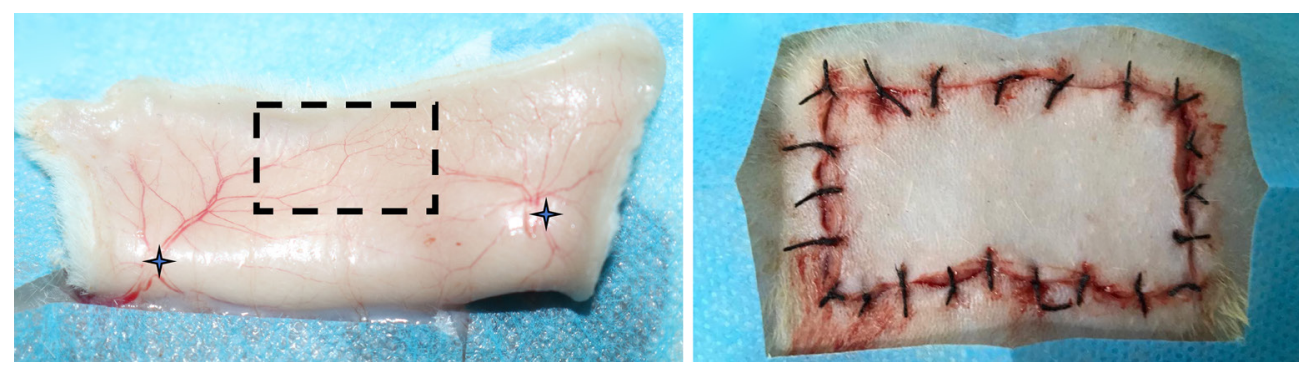

Figure 3 Multi-territory perforator flap outlining. Establishment multiterritory perforator flap animal models, including 2 vascular territories and 1 choke zone, were based on the deep circumflex iliac artery with a minimal cuff of muscle surrounding the perforator under the direction of the PAM image. The dotted line indicates the choke zone. The black arrow indicates the deep circumflex iliac perforator and intercostal perforator. 
Table 1 Distribution of perforators in the backs of mice $(n=7)$

\begin{tabular}{lccc}
\hline Name of the perforators & Diameter of the perforating artery and vein $(\mathrm{mm})$ & Area of the perforasome $\left(\mathrm{mm}^{2}\right)$ & Distribution/total area $(\%)$ \\
\hline Intercostal perforator & $0.43 \pm 0.02 / 0.14 \pm 0.01$ & $237.72 \pm 6.64$ & $22.11 \pm 0.36$ \\
Deep circumflex perforator & $0.56 \pm 0.04 / 0.15 \pm 0.01$ & $475.56 \pm 7.13$ & $44.29 \pm 0.30$ \\
\hline
\end{tabular}

imaging acquisition was conducted preoperatively and at 6,12 , and 18 hours postoperatively. The "dark zone" with hardly any intact microvessels in PAM images was counted as a prediction of necrosis. After 18 hours of observation, every mouse was raised in a separated cage in an air-conditioned room and fed a standard laboratory diet for 7 days. The overall 7-day duration was an adequate amount of time to exhibit the clinical necrosis of flap tissue. Digital photographs were acquired before surgery and on postoperative day 7 to demonstrate flap necrosis. When the skin was black or an eschar formed, it was defined as flap necrosis. For the purpose of drawing the accurate boundary of flap necrosis, 3 clinical experts (who were blinded to the results of PAM images) assessed flap necrosis via digital photographs and clinical assessment on the first 7 postoperative days and outlined the boundary between the viable and necrotic areas of the flap. In addition, in order to observe the vascular morphological changes of the choke region, the choke zone was measured using PAM at specified time: before surgery; immediately after surgery; and at 2, 4, and 6 days postoperatively.

\section{Statistical analysis}

The original data were used to obtain PAM images via our developed algorithm implemented in LabVIEW (2016, National Instruments, USA), and image postprocessing was then performed using MATLAB (R2014b, the MathWorks, USA). ImageJ (National Institutes of Health, USA) software was used for calculation of the percentage area of the dark zone in PAM images and the percentage area of clinical necrotic area in the digital image. Vascular quantification was analyzed via ImageJ. Vascular density, tortuosity, and photoacoustic intensity were computed to accurately describe the changes in vascular properties. All data are expressed as mean $\pm \mathrm{SD}$.

\section{Results}

\section{Preoperative perforator mapping in the mouse back using PAM imaging}

Preoperative perforator mapping visualized the detailed information concerning perforators in the semi-back of mice. PAM images showed there were 2 perforators in total, known as the intercostal perforator and the deep circumflex perforator, and accurately identified the location of each perforator in real time (Figure $2 F$ ). More importantly, the PAM images also clearly displayed the microvascular boundary of each perforator, which permitted precise planning of the flap boundaries (Figure 2B,C). As is shown in Table 1, graphical analysis showed that the intercostal arterial/venous diameters were $0.14 \pm 0.01$ and $0.43 \pm 0.02 \mathrm{~mm}$, and the area of the intercostal perforasomes were $237.72 \pm 6.64 \mathrm{~mm}^{2}$. Also, the deep circumflex arterial/venous diameters were $0.15 \pm 0.01$ and $0.56 \pm 0.04 \mathrm{~mm}$, and the area of the deep circumflex perforasomes were $475.56 \pm 7.13 \mathrm{~mm}^{2}$. The results also demonstrated that the deep circumflex perforator was the predominant perforator in the mouse back and has a larger distribution than does the intercostal perforator (Table 1).

\section{Postoperative quantitative assessment of flap status via PAM imaging}

A multiterritory perforator dorsal flap was designed and harvested based on the deep circumflex iliac perforator, which included 2 perforasomes (Figure 3). The results showed that all multiterritory perforator flaps exhibited different levels of necrosis at the end of the observation time. In addition, the dark zone with impaired microcirculation in PAM images, which continuously extends over time, was a predictor of the necrotic area in flaps, which was similar at 18 hours postoperatively to the area of clinical necrosis on postoperative day 7. Figure 4 displays a representative region of predicted necrosis and clinical necrosis on postoperative day 7 drawn with the PAM images.

We then compared these drawn dark areas in the PMA images at every specified time points to the drawn postoperative day 7 necrotic areas. The full flap size always changed over time due to slight shifts in camera positions and tissue healing contracture. We expressed the dark zone in the PAM images and clinical necrotic area of the flap as 


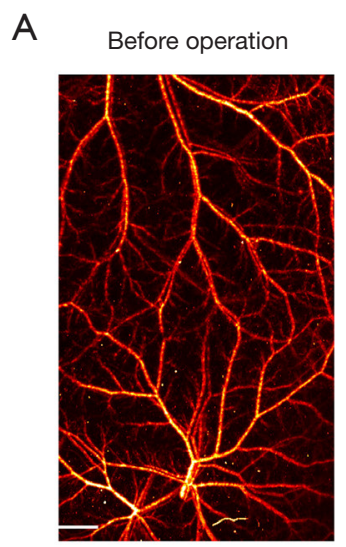

B
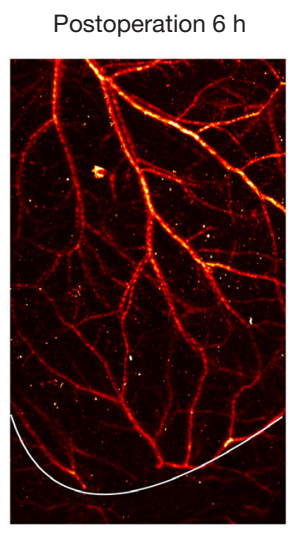

E

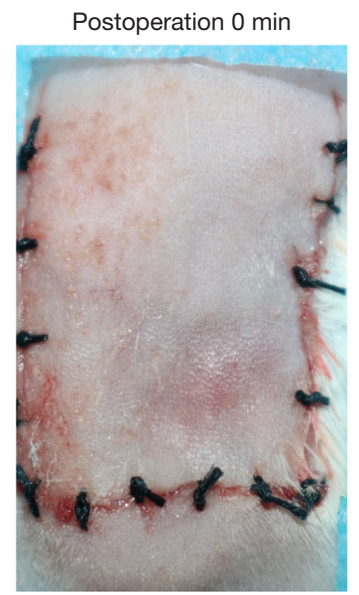

C

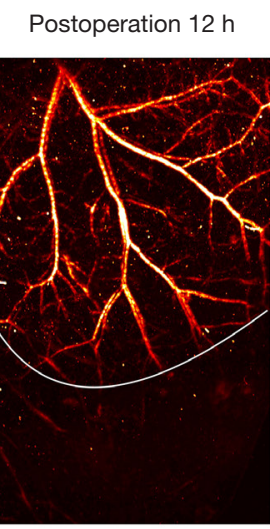

\section{$\mathrm{F}$}

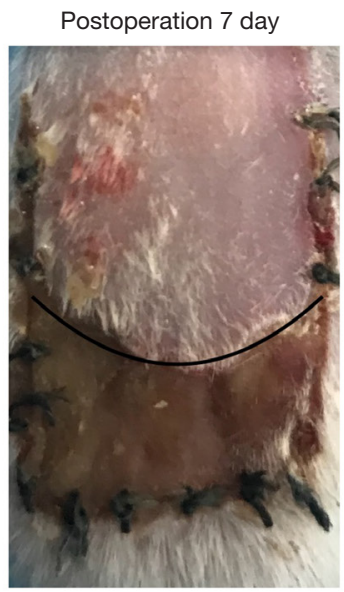

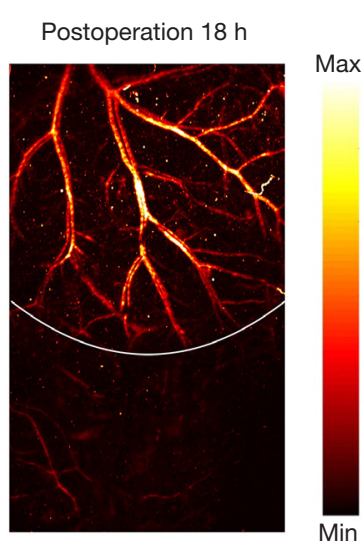

Figure 4 Imaging of multiterritory perforator flap. (A,B,C,D) PAM imaging results at different points. (E,F) The digital images at immediately after the injury and on day 7 postoperatively.

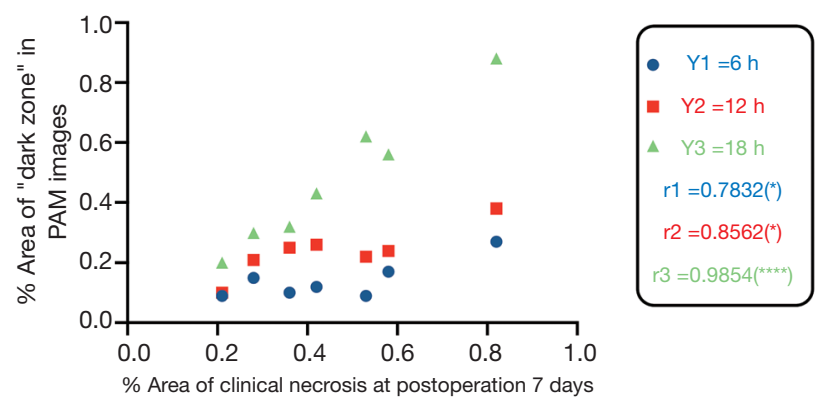

Figure 5 Pearson correlation demonstrated a linear relationship between the percentage area of predicted necrosis by the PAM image at 6,12 , and 18 hours postoperatively and the percentage area of clinical necrosis on postoperative day 7 . relative percentages compared to the total flap size for each time point. Linear comparisons were subsequently made between the dark zone percentage area of flaps measured at 6,12 , and 18 hours postoperatively via PAM and the percentage area of clinical necrosis of flaps on postoperative day 7. As shown in Figure 5, there was a strong positive linear correlation between the predictive necrosis in PAM images at 6 hours and 12 hours after surgery, and the clinical necrotic area on postoperative day $7(\mathrm{r}=0.783, \mathrm{P}=0.037$; $\mathrm{r}=0.856, \mathrm{P}=0.0139$ ), and a stronger linear correlation between the predictive necrosis in PAM images at 18 hours after surgery and the clinical necrotic area on postoperative day $7(\mathrm{r}=0.985, \mathrm{P}<0.0001)$. 
A

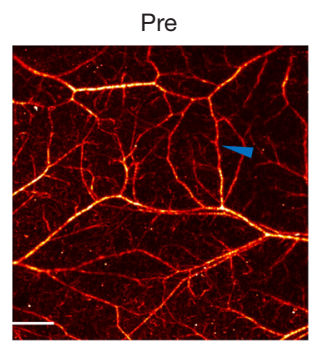

B

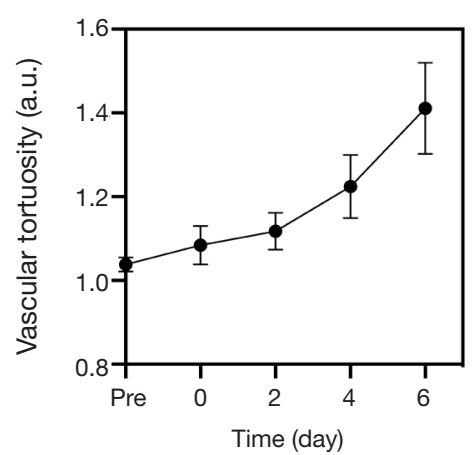

0 day

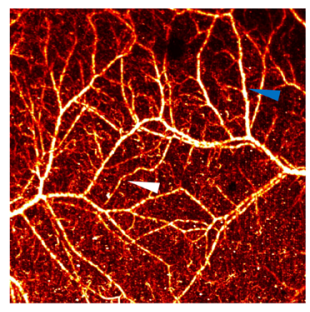

2 day

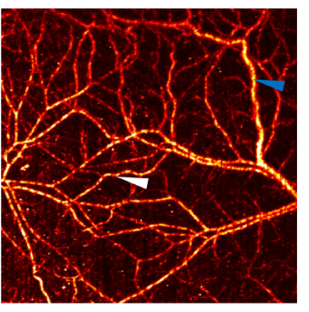

C

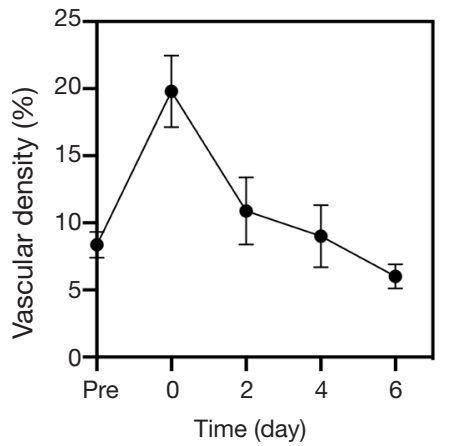

4 day

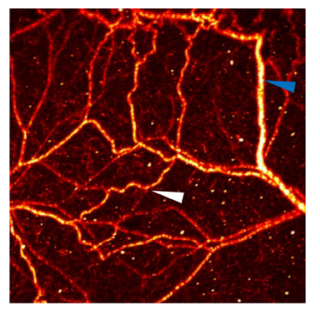

6 day

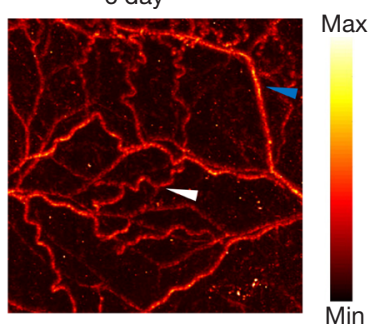

D

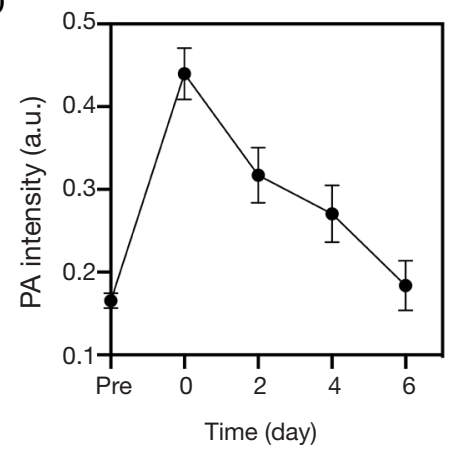

Figure 6 Representative PAM images of choke zone in the flap which occurred distal necrosis at four special time points : before the flap was harvested, immediately after the flap harvested, 2, 4, 6 days (A). The white triangle indicates the choke vessel which opened immediately after the flap harvested, and the blue triangle indicates the vessel which the vascular tortuosity and PA intensity changed significantly. (B,C,D) Quantitative analysis of vascular tortuosity, vascular tortuosity and PA intensity detected via PAM in choke zone.

\section{PAM imaging for observations of the choke zone in the multiterritory perforator skin flap model}

Determining the changes of choke vessels is an important target for studying multiterritory flaps and plays an essential role in basic studies of flap survival. As mentioned above, all 7 mice involved in this part of the study exhibited distal necrosis. The decision about flap viability was made by 3 experienced surgeons based on the PAM images and clinical assessment at the end of observation time. All flaps survived, and distal partial necrosis was seen. Using the PAM image system, the vessels in the choke zone could be observed clearly during the entire course of the experiment. As shown in Figure 6A, the PAM images of the choke zone were obtained before the flap was harvested, immediately after surgery, and on the second, fourth, and sixth days after the flap was harvested. To gain quantitative insight into the changes of vessels in the choke zone, the PA intensity, vascular density, and vascular tortuosity were calculated before and after the flap was obtained (Figure 6B,C,D). The analysis showed that vascular tortuosity increased significantly over time compared to the baseline of vessels in the choke zone, indicating increased vascular resistance.
Vascular tortuosity peaked on the sixth day after the flap was harvested; however, the PA intensity and vascular density peaked immediately after surgery, but waned over time. In addition, the PAM could accurately show that the choke vessels opened immediately after the flap was harvested.

\section{Discussion}

Among the various surgical techniques to conceal large and complex defects, the perforator flap is particularly prevalent in reconstructive microsurgery due to its optimal reconstruction with minimum donor-site morbidity $(24,25)$. Having a comprehensive understanding of cutaneous microvessels is key to the design and clinical use of perforator skin flaps. Considering its invasiveness and poor resolution, $\mathrm{X}$-ray has been regarded as an ineffective approach for detecting the perforator. Over the last decade, CTA and MRA have been considered the gold standard in many situations (12). Both of these techniques can accurately detect the number, location, caliber, and performance of perforators. However, they also have some obvious disadvantages. For example, 
they require the use intravenous contrast, which cannot be used in patients with renal impairment or severe claustrophobia. Additionally, they involve high costs, have a limited observation window, expose patients to radiation, and can fail to accurately visualize the perforasome of individual perforators due to their limited resolutions. Meanwhile, infrared thermography, which has been described previously by researchers (26), can automatically and reliably detect the perforators in thermograms, but the detailed information produced cannot be clearly visualize. Moreover, photoacoustic tomography, as a branch of photoacoustic image technology, was previously used to identify the location of the anterolateral thigh perforator in clinical study; however, due to its poor resolution and contrast, it is difficult to visualize very small perforators or the clear boundary of perforators, which can significantly limit the perforator flap design. As a new noninvasive imaging and analysis technology, PAM, which facilitates obtaining vascular structure (especially for microvessels) in a comprehensive and quantitative way, has attracted considerable attention. The core advantage of PAM is its high resolution and contrast of microvessels. Recently, increasing evidence has shown that PAM is widely used in various basic and clinical studies due to this particular advantage. For example, PAM was used in structural and functional brain imaging to explore cerebral small vessel diseases, including brain tumor, stroke, Alzheimer disease, and ischemic neurodegeneration in small animals. Furthermore, it has been used in thyroid and breast imaging, has the potential to detect more microvessels than does color Doppler, and has been demonstrated to be able to distinguish between microvessel nerve complexes and surrounding tissue intraoperatively in gynecological and urologic disorders, thus markedly improving surgical outcomes. Although PAM exhibits excellent performance in mapping microvessels, there are no studies involving the application of PAM in the perforator flap design. In our study, we showed that PAM can provide detailed information including the number, location, boundary, and caliber of perforators in vivo in real time with high spatial and temporal resolution. This allows surgeons to design complex flaps more easily by facilitating perforator flap design, reducing operating time, and improving the flap survival rate.

After dissection of the perforator flap, noninvasively and accurately assessing the perforator flap status remains a challenge for both surgeons and patients. Previous studies have shown that photoacoustic imaging offers great potential for monitoring the tissue status by noninvasively exploiting the optical absorption of blood hemoglobin (27). It has been used to assess the status of burn healing, monitoring angiogenesis of tumors, visualizing dynamic changes of vessels in cerebral ischemia and reperfusion models, and evaluating photodynamic therapy in animals (18,28-31). In our study, we used the PAM system at 6, 12, and 18 hours after surgery to predict flap survival, and our data demonstrated a significant positive correlation between our predictions and the clinical outcome provided by 3 experienced surgeons on the seventh day after surgery. The results also showed that the predictive necrosis outlined by the PAM system at 18 hours postoperatively had a stronger linear correlation with the ultimate necrotic area. Therefore, our findings suggest that the PAM imaging method is an effective tool for the early and quantitative assessment of flap status by outlining accurate potential boundaries of flap necrosis, and can guide surgeons in providing surgical intervention as promptly as possible, thus improving the flap survival rate after surgery.

Another research priority concerning the perforator involved examining the microcirculation of choke vessels, which play an essential role in multiterritory perforator flap survival. Studies have reported that one perforator vessel can perfuse an adjacent territory via choke vessels (32). Researchers have further developed this hypothesis, proposing a concept of "watershed" for describing the importance of choke vessels in adjoining vascular territories (33). It is generally agreed upon that the key mechanism for obtaining a pedicle perforator flap that is oversized for its territory should be based on the behavior of choke vessels. To date, various methods including platelet rich plasma, leonurine, and flap surgical delay technology have been attempted to influence the behavior of choke vessels to promote multiterritory perforator flap survival (9,34-36).

However, in the past few years, there has been a lack of available methods to facilitate the analysis of vessel morphology and morphometry, which has significantly hindered the study of choke vessels. Compared to traditional radiative imaging modalities that are used for morphological analysis of choke vessels, such as X-ray angiography and CTA, the PAM imaging modality benefits from its high safety with nonradiation, continuous observation in vivo over a long period, high resolution, and deep penetration, which can facilitate the visualization and quantitative analysis of choke vessels. In our study, the PAM imaging modality was used to quantitatively visualize the parameters of choke vessels, such as PA intensity, vascular 
density, and vascular tortuosity, and also clearly showed the opening of the choke vessels across territories after the formation of a perforator flap.

\section{Conclusions}

In summary, this study explicitly showed that PAM can be used as an effective imaging technology platform for the clinical application and scientific research of perforator flaps, including visualizing perforator mapping, predicting the potential necrosis zone of the flap, and observing morphological changes of the choke vessel in a noninvasive and dynamic way.

\section{Acknowledgments}

Funding: This work was supported by the Health Commission of Hubei Province Medical Leading Talent Project (no. LJ20200405) and the National Natural Science Foundation of China (no. 82072440).

\section{Footnote}

Conflicts of Interest: All authors have completed the ICMJE uniform disclosure form (available at https://dx.doi. org/10.21037/qims-21-135). The authors have no conflicts of interest to declare.

Ethical Statement: The authors are accountable for all aspects of the work in ensuring that questions related to the accuracy or integrity of any part of the work are appropriately investigated and resolved. All of the experimental procedures conformed to the institutional guidelines for the care and use of animals to minimize discomfort to the animals during the surgery and recovery periods, and experiments were performed under a project license (no. 2019173) granted by the Committee on the Ethics of Animal Experiments of Wuhan University.

Open Access Statement: This is an Open Access article distributed in accordance with the Creative Commons Attribution-NonCommercial-NoDerivs 4.0 International License (CC BY-NC-ND 4.0), which permits the noncommercial replication and distribution of the article with the strict proviso that no changes or edits are made and the original work is properly cited (including links to both the formal publication through the relevant DOI and the license). See: https://creativecommons.org/licenses/by-nc-nd/4.0/.

\section{References}

1. Tenekeci G. Perforator Flaps: Principles and Techniques. Issues in Flap Surgery. London, UK: IntechOpen, 2018.

2. Taylor GI, Corlett RJ, Dhar SC, Ashton MW. The anatomical (angiosome) and clinical territories of cutaneous perforating arteries: development of the concept and designing safe flaps. Plast Reconstr Surg 2011;127:1447-59.

3. Chen SH, Xu DC, Tang ML, Ding HM, Sheng WC, Peng TH. Measurement and analysis of the perforator arteries in upper extremity for the flap design. Surg Radiol Anat 2009;31:687-93.

4. Taylor GI, Corlett RJ, Ashton MW. The Functional Angiosome: Clinical Implications of the Anatomical Concept. Plast Reconstr Surg 2017;140:721-33.

5. Vargas CR, Iorio ML, Lee BT. A Systematic Review of Topical Vasodilators for the Treatment of Intraoperative Vasospasm in Reconstructive Microsurgery. Plast Reconstr Surg 2015;136:411-22.

6. Suszynski TM, Haddock NT, Teotia SS. Expanding the Use of the Perforator Free Flap in Breast Reconstructive and Aesthetic Surgery. Plast Reconstr Surg 2019;143:900e-1e.

7. Luo Z, Wu P, Qing L, Zhou Z, Yu F, Zhang P, Tang J. The hemodynamic and molecular mechanism study on the choke vessels in the multi-territory perforator flap transforming into true anastomosis. Gene 2019;687:99-108.

8. Low OW, Sebastin SJ, Cheah AEJ. A Review of Pedicled Perforator Flaps for Reconstruction of the Soft Tissue Defects of the Leg and Foot. Indian J Plast Surg 2019;52:26-36.

9. Lin D, Wu H, Zhou Z, Tao Z, Gao W, Jia T. The Effect of Leonurine on Multiterritory Perforator Flap Survival in Rats. J Surg Res 2020;245:453-60.

10. Abdelfattah U, Power HA, Song S, Min K, Suh HP, Hong JP. Algorithm for Free Perforator Flap Selection in Lower Extremity Reconstruction Based on 563 Cases. Plast Reconstr Surg 2019;144:1202-13.

11. Muntean MV, Strilciuc S, Ardelean F, Pestean C, Lacatus R, Badea AF, Georgescu A. Using dynamic infrared thermography to optimize color Doppler ultrasound mapping of cutaneous perforators. Med Ultrason 2015;17:503-8.

12. Nie JY, Lu LJ, Gong X, Li Q, Nie JJ. Delineating the vascular territory (perforasome) of a perforator in the lower extremity of the rabbit with four-dimensional computed tomographic angiography. Plast Reconstr Surg 2013;131:565-71.

13. Lee JW, Kim HK, Kim SR, Han YS, Park JH. Preoperative 
identification of a perforator using computed tomography angiography and metal clip marking in perforator flap reconstruction. Arch Plast Surg 2015;42:78-83.

14. Azuma R, Morimoto Y, Masumoto K, Nambu M, Takikawa M, Yanagibayashi S, Yamamoto N, Kikuchi M, Kiyosawa T. Detection of skin perforators by indocyanine green fluorescence nearly infrared angiography. Plast Reconstr Surg 2008;122:1062-7.

15. Qin W, Jin T, Guo H, Xi L. Large-field-of-view optical resolution photoacoustic microscopy. Opt Express 2018;26:4271-8.

16. Wang Y, Xing D, Zeng Y, Chen Q. Photoacoustic imaging with deconvolution algorithm. Phys Med Biol 2004;49:3117-24.

17. Zhang W, Ma H, Cheng Z, Wang Z, Zhang L, Yang S. Miniaturized photoacoustic probe for in vivo imaging of subcutaneous microvessels within human skin. Quant Imaging Med Surg 2019;9:807-14.

18. Yang F, Wang Z, Zhang W, Ma H, Cheng Z, Gu Y, Qiu $\mathrm{H}$, Yang S. Wide-field monitoring and real-time local recording of microvascular networks on small animals with a dual-raster-scanned photoacoustic microscope. J Biophotonics 2020;13:e202000022.

19. Yao J, Wang LV. Photoacoustic microscopy. Laser \& Photonics Reviews 2013;7:758-78.

20. Wang Z, Yang F, Ma H, Cheng Z, Yang S. Photoacoustic and ultrasound (PAUS) dermoscope with high sensitivity and penetration depth by using a bimorph transducer. J Biophotonics 2020;13:e202000145.

21. Liu C, Liao J, Chen L, Chen J, Ding R, Gong X, Cui C, Pang Z, Zheng W, Song L. The integrated high-resolution reflection-mode photoacoustic and fluorescence confocal microscopy. Photoacoustics 2019;14:12-8.

22. Yang J, Zhang G, Li Q, Liao C, Huang L, Ke T, Jiang H, Han D. Photoacoustic imaging for the evaluation of early tumor response to antivascular treatment. Quant Imaging Med Surg 2019;9:160-70.

23. Laser Institute of America. American National Standard for Safe Use of Lasers ANSI Z136.1-2014. American National Standards Institute, Inc., 2014.

24. Tuinder SMH, Beugels J, Lataster A, de Haan MW, Piatkowski A, Saint-Cyr M, van der Hulst R, Allen RJ. The Lateral Thigh Perforator Flap for Autologous Breast Reconstruction: A Prospective Analysis of 138 Flaps. Plast Reconstr Surg 2018;141:257-68.

25. Kim JT, Kim SW. Perforator Flap versus Conventional Flap. J Korean Med Sci 2015;30:514-22.

26. Tsuge I, Saito S, Yamamoto G, Sekiguchi H, Yoshikawa
A, Matsumoto Y, Suzuki S, Toi M. Preoperative vascular mapping for anterolateral thigh flap surgeries: A clinical trial of photoacoustic tomography imaging. Microsurgery 2020;40:324-30.

27. Xiong Y, Chen L, Yan C, Zhou W, Yu T, Sun Y, Cao F, Xue H, Hu Y, Chen D, Mi B, Liu G. M2 Macrophagy-derived exosomal miRNA-5106 induces bone mesenchymal stem cells towards osteoblastic fate by targeting salt-inducible kinase 2 and 3. J Nanobiotechnology 2020;18:66.

28. Lu W, Huang Q, Ku G, Wen X, Zhou M, Guzatov D, Brecht P, Su R, Oraevsky A, Wang LV, Li C. Photoacoustic imaging of living mouse brain vasculature using hollow gold nanospheres. Biomaterials 2010;31:2617-26.

29. Yang S, Xing D, Lao Y, Yang D, Zeng L, Xiang L, Chen WR. Noninvasive monitoring of traumatic brain injury and posttraumatic rehabilitation with laser-induced photoacoustic imaging. Applied Physics Letters 2007;90:629.

30. Wu Z, Duan F, Zhang J, Li S, Ma H, Nie L. In vivo dualscale photoacoustic surveillance and assessment of burn healing. Biomed Opt Express 2019;10:3425-33.

31. Oraevsky AA, Clingman B, Zalev J, Stavros AT, Yang WT, Parikh JR. Clinical optoacoustic imaging combined with ultrasound for coregistered functional and anatomical mapping of breast tumors. Photoacoustics 2018;12:30-45.

32. Zhuang Y, Xie Y, Wu Z, Xue L, Tang Y, Zheng H, Fang F. Hemodynamic Study of Three Patterns of Flaps on Rats with a Novel Panoramic Photographing Technique Involved. Plast Reconstr Surg 2016;138:653e-65e.

33. Zhuang Y, Hu S, Wu D, Tang M, Xu DC. A novel in vivo technique for observations of choke vessels in a rat skin flap model. Plast Reconstr Surg 2012;130:308-17.

34. Mao Y, Li H, Ding M, Hao X, Pan J, Tang M, Chen S. Comparative Study of Choke Vessel Reconstruction With Single and Multiple Perforator-Based Flaps on the Murine Back Using Delayed Surgery. Ann Plast Surg 2019;82:93-8.

35. Jiang Z, Li X, Chen M, Lu L, Gong X. Effect of Endogenous Vascular Endothelial Growth Factor on Flap Surgical Delay in a Rat Flap Model. Plast Reconstr Surg 2019;143:126-35.

36. Chai J, Ge J, Zou J. Effect of Autologous PlateletRich Plasma Gel on Skin Flap Survival. Med Sci Monit 2019;25:1611-20.

Cite this article as: Zhang D, Chen H, Hu X, Yu A. Photoacoustic microscopy: a novel approach for studying perforator skin flap in a mouse model. Quant Imaging Med Surg 2021;11(10):4365-4374. doi: 10.21037/qims-21-135 